\title{
Hard X-ray In-situ Full-field Microscopy for Material Science Applications.
}

$\underline{\text { Irina Snigireva }}^{1 *}$, Ken Vidar Falch ${ }^{2}$, Daniele Casari ${ }^{2}$, Marco Di Michiel ${ }^{1}$, Carsten Detlefs ${ }^{1}$, Ragnvald Mathiesen $^{2}$, and Anatoly Snigirev ${ }^{3}$

1. European Synchrotron Radiation Facility, 38000 Grenoble, France

2. Department of Physics, Norwegian University of Science and Technology, 7491 Trondheim, Norway

3. Immanuel Kant Baltic Federal University, 238300 Kaliningrad, Russia

* Corresponding author, email: irina@esrf.fr

Hard X-ray transmission microscopy (HXTM) based on refractive X-ray optics was employed as an instrument in material science to investigate buried-in microstructures in two or three dimensions with spatial resolution approaching $100 \mathrm{~nm}$ [1-4]. In this microscope, the incoming beam goes through the condenser, and illuminates the sample. The objective lens magnifies the image of the sample on the highresolution CCD camera (pixel size $0.65 \mu \mathrm{m}$ ). The X-ray magnification typically lies in the 5-100 range but is not strictly limited. With the extra magnification from X-ray optics, the maximum spatial resolution is no longer limited by the detector pixel size, but by the X-ray optics. The main factors influencing the spatial resolution are the numerical aperture and aberrations of objective lens.
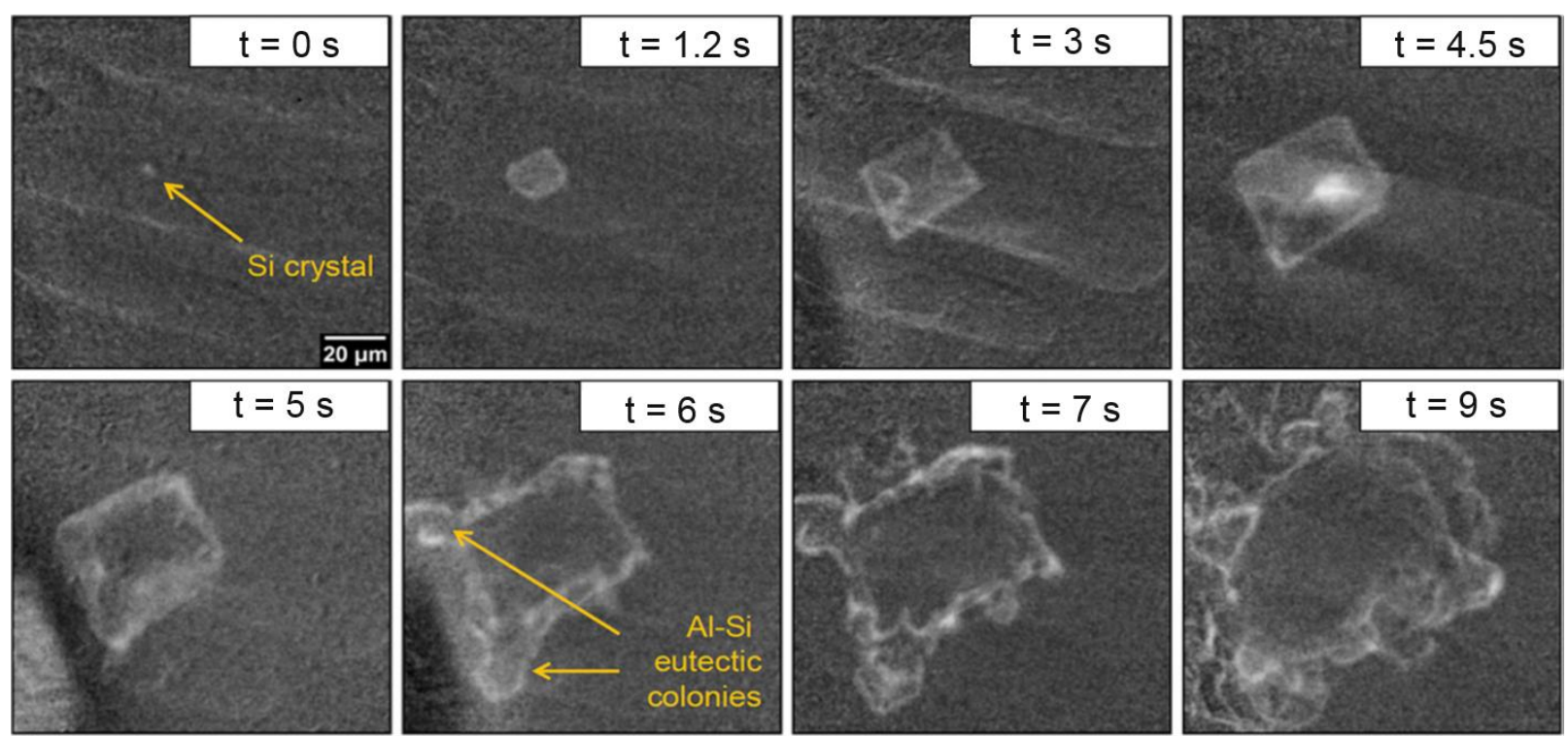

Figure. 1. Sr-modified irregular eutectic microstructure formation of Al-16.4Cu-7.1Si Sr modified alloy. $t$ values given in the upper right of each image is the time at which the Si crystal was observed in the field of view. The cooling rate was $0.1 \mathrm{~K} / \mathrm{s}$, magnification 10x, effective pixel size $125 \mathrm{~nm}$, exposure $0.3 \mathrm{~s}$ [5].

The HXTM was applied for in-situ studies of eutectic phase transformations in metallic alloys. The image in Fig. 1 was obtained with $17 \mathrm{keV}$ monochromatic radiation, and captures the nucleation and grows of a facetted Si crystal in a highly hypo-eutectic Al-16.4Cu-7.1Si with $5 \mathrm{ppm} \mathrm{P}$ and $100 \mathrm{ppm} \mathrm{Sr}$ added to provide nucleation sites for $\mathrm{Si}$ and to yield eutectic modification, respectively. The resolution of HXTM permits to identify the Si crystal at an early growth stage based on its octahedral growth morphology, allowing detailed study how branching evolves from the primary Si crystal. After nucleation and several seconds of Si crystal growth, instabilities and structures are observed to evolve starting from two vertices and then from the edges of the crystal, which act as substrates to formation of Al-Si eutectic colonies [5]. 
Applying a special illumination scheme that works effectively to reduce chromatic aberrations, the HXTM can operate in non-monochromatic beam [6]. Switching to broader bandwidth, frame rates down to a few milliseconds can be realized, opening new possibilities for in situ studies of microstructure evolution and response to external fields at spatiotemporal resolutions that go well beyond previous benchmarks. Figure 6 shows a slice of a reconstructed tomogram of a solidified $\mathrm{Al}-\mathrm{Al} 2 \mathrm{Cu}$ eutectic microstructure. The tomogram was constructed from 900 projections, each recorded with $2 \mathrm{~ms}$ exposure time, making the total acquisition time $1.8 \mathrm{~s}$, and spanning a volume of $\sim 200 \times 200 \times 200 \mu \mathrm{m}^{3}$. The lamella spacing is observed to vary markedly across the field of view, where the smallest lamella half-period measured was $270 \mathrm{~nm}$. The magnification of the X-ray image was 8 while the magnification of the visible-light optics was 10 .

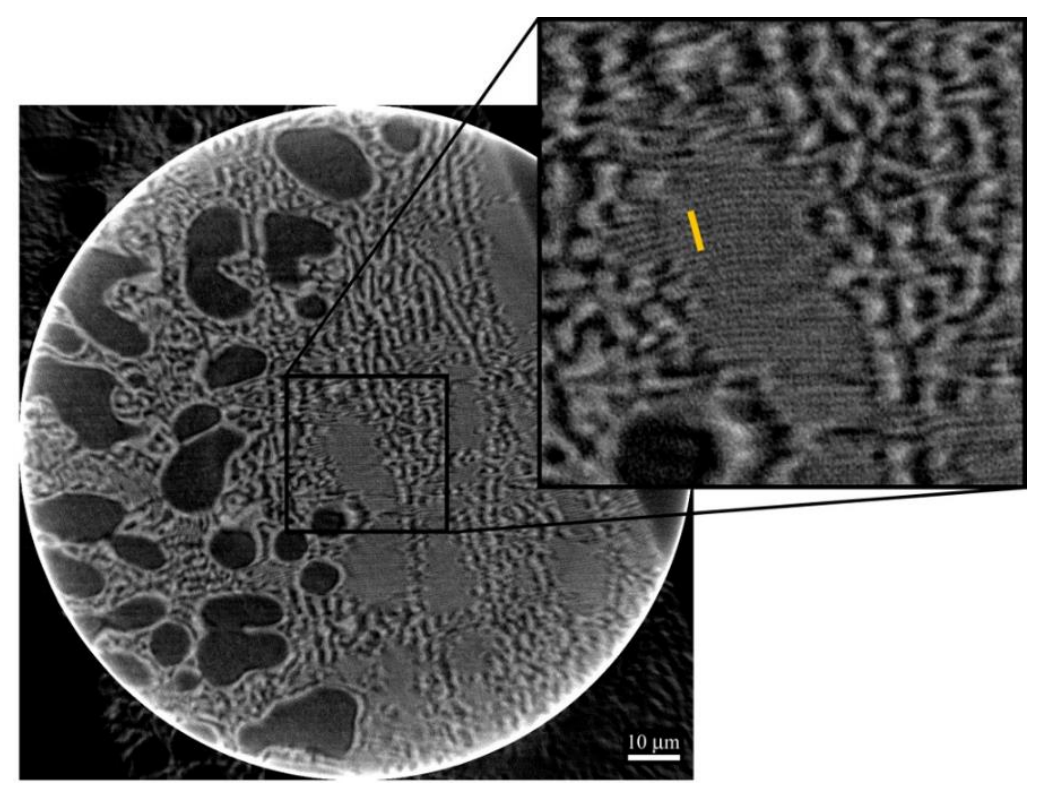

Figure. 2. Reconstructed tomogram slice of $\mathrm{Al}-\mathrm{Al} 2 \mathrm{Cu}$ eutectic microstructure recorded using $17 \mathrm{keV}$. Large dark pockets are $\alpha$-Al primary dendrites, while dark and bright areas inside the eutectic are the Al phase and the $\mathrm{A} 12 \mathrm{Cu}$ phase, respectively. The orange line in the zoomed view indicates where the smallest lamellae half-period of $270 \mathrm{~nm}$ was measured. The pixel size is $125 \mathrm{~nm}$ [5].

The Zernike phase contrast (ZPC) for X-ray microscopy based on compound refractive lenses was successfully realized at photon energies above $17 \mathrm{keV}$ [7]. Phase contrast was achieved by fitting a CRL with a circular phase plate. The sub-micrometer resolution has been demonstrated which can be improved using already existing technology. The possibility of combining ZPC imaging with polychromatic radiation was considered, and a preliminary test experiment was performed with positive results.

\section{References:}

[1] A. Snigirev, V. Kohn, I. Snigireva, B. Lengeler, Nature 384 (1996) 49-51.

[2] V. Kohn, I. Snigireva, A. Snigirev, Opt. Comm. 216 (2003) 247-260.

[3] A. Bosak, I. Snigireva, K. Napolskii, A. Snigirev, Adv. Mater. 22 (2010) 3256-3259.

[4] D. Byelov et al, RSC Advances 3 (2013) 15670.

[5] K. V. Falch, et al, J. Mater Sci. 52 (2017) 3497-3507.

[6] K. V. Falch, et al, Appl. Phys. Lett. 109 (2016) 054103.

[7] K. V. Falch, et al, Ultramicroscopy 184 (2018) 267-273. 